Article

\title{
Random pullback attractor of a non-autonomous local modified stochastic Swift-Hohenberg with multiplicative noise
}

\author{
Yongjun $\mathrm{Li}^{{ }^{*}}{ }$, Tinggang $\mathrm{Zhao}^{1}$ and Hongqing $\mathrm{Wu}^{1}$ \\ 1 School of Mathematics, Lanzhou City University, Lanzhou, 730070, P.R. China; li_liyong120@163.com (Y. Li); \\ 13669397938@163.com(T.Zhao); wuhq@lzcu.edu.cn(H.Wu), \\ * Correspondence: li_liyong120@163.com (Y. Li)
}

\begin{abstract}
In this paper, we study the existence of the random $\mathcal{D}$-pullback attractor of a non-autonomous local modified stochastic Swift-Hohenberg equation with multiplicative noise in stratonovich sense. It is shown that a random $\mathcal{D}$-pullback attractor exists in $\mathrm{H}_{0}^{2}(D)$ when its external force has exponential growth. Due to the stochastic term, the estimate are delicate, we overcome this difficulty by using the Ornstein-Uhlenbeck $(\mathrm{O}-\mathrm{U})$ transformation and its properties.
\end{abstract}

Keywords: Swift-Hohenberg equation; Random $\mathcal{D}$-pullback attractor; Non-autonomous random dynamical system

\section{Introduction}

The Swift-Hohenberg(S-H) type equations arise in the study of convective hydrodynamical, plasma confinement in toroidal and viscous film flow, was introduced by authors in [1]. After that, Doelman and Standstede [2] proposed the following modified Swift-Hohenberg equation for a pattern formation system near the onset to instability

$$
u_{t}+\Delta^{2} u+2 \Delta u+a u+b|\nabla u|^{2}+u^{3}=0
$$

where $\mathrm{a}$ and $\mathrm{b}$ are arbitrary constants.

We can see from the above equation that there exist two operators $\Delta$ and $\Delta^{2}$, these two operators have some symmetry, for any $u \in H_{0}^{1}(D)$, the inner product $(\Delta u, u)=-(\nabla u, \nabla u)=\|\nabla u\|^{2}$, for any $u \in H_{0}^{2}(D), \quad\left(\Delta^{2} u, u\right)=(\Delta u, \Delta u)=\|\Delta u\|^{2}, \Delta$ is antisymmetry and $\Delta^{2}$ is symmetry. We will use the symmetry principle study S-H equation.

The dynamical properties of the S-H equation are important for the studies pattern formation system have been extensively investigated by many authors; see [3-8]. Polat [8] establish the existence of global attractor for the system (1.1), and then Song et al. [7] improved the result in $\mathrm{H}^{k}$.

Recently for non-autonomous modified S-H equation:

$$
d u+\left(\Delta^{2} u+2 \Delta u+a u+b|\nabla u|^{2}+u^{3}-g(x, t)\right) d t=l u \circ d W(t),
$$

it has also attracted the interest of many authors. If $l=0$, equation (1.2) becomes a non-autonomous modified S-H equaiton. Park [9] proved the existence of $\mathcal{D}$-pullback attractor when the external force has exponential growth, $\mathrm{Xu}$ et al.[10] established the existence of uniform attractor when the external force $g(x, t)$ satisfies translation bounded, these results need the spatial variable in two dimensions. When $l=0$, equation (1.2) becomes a non-autonomous stochastic S-H equation, if $|b|<<1$ is a constant, Guo et al.[11] investigated the equation when $g(x, t)=0$ and proved the existence of random attractor which need the spatial variable in one dimension. For $g(x, t)=0$, to the best of our knowledge, the existence of random $\mathcal{D}$-pullback attractor for equation (1.2) has not yet considered. 
In this paper, we consider the following one dimensional non-autonomous local modified stochastic S-H equation with multiplicative noise:

$$
\begin{aligned}
& d u+\left(\Delta^{2} u+2 u_{x x}+a u+b u_{x}^{2}+u^{3}-g(x, t)\right) d t=l u \circ d W(t), \text { in } D \times[\tau, \infty), \\
& u=u_{x x}=0, x \in \partial D, \\
& u(x, \tau)=u_{\tau}, x \in D
\end{aligned}
$$

Where $D$ is a bounded open interval, $\Delta u$ means $u_{x x}$, and $\Delta^{2} u$ means $u_{x x x x},|b|<4, a$ and $l$ are arbitrary constants, $W(t)$ is a two-sided real-valued Wiener process on a probability space which will be specified later. For the external force $g(x) \in L_{l o c}^{2}\left(R, L^{2}(D)\right)$, we assume that there exist $M>0$ and $\beta>0$ such that

$$
a-\beta-5<0, \quad\|g(x, t)\|^{2} \leq M e^{\gamma|t|}, \text { for any } t \in \mathbb{R}, \quad 0 \leq \gamma<\frac{3 \beta}{11} .
$$

The assumption is same as $[8,12]$, through simple calculation, for all $t \in R$, we have

$$
H(t):=\int_{-\infty}^{t} e^{\beta s}\|g(x, s)\|^{2} d s<\infty, \quad \int_{-\infty}^{t} e^{-\frac{8 \beta}{3} s} H^{\frac{11}{3}}(s) d s<\infty .
$$

An outline of this paper is as follows: In section 2, we recall some basic concepts about random $\mathcal{D}$-pullback attractors. In Section 3, we prove that the stochastic dynamical system generated by (1.3) exists a random $\mathcal{D}$-pullback attractor in $H_{0}^{2}(D)$.

\subsection{Preliminaries}

There are many research results on random attractors and related issues. The reader is referred to [13-19] for more details, we only list the definitions and abstract result

Let $\left(X,\|\cdot\|_{X}\right)$ be a separable Banach space with Borel $\sigma$-algebra $\mathcal{B}(X)$ and $(\Omega, \mathcal{F}, \mathbb{P})$ be a probability space. In this paper, the term $\mathbb{P}$-a.s.(the abbreviation for $\mathbb{P}$ almost surely) denotes that an event happens with probability one. In other words, the set of possible exception may be non-empty, but it has probability zero.

Definition 2.1.([15,16,20,21]) $\left(\Omega, \mathcal{F}, \mathbb{P},\left(\theta_{t}\right)_{t \in \mathbb{R}}\right)$ is called a metric dynamical systems if $\theta: \mathbb{R} \times \Omega \rightarrow \Omega$ is $(\mathcal{B}(\mathbb{R}) \otimes \mathcal{F}, \mathcal{F})$-measurable, and $\theta_{0}$ is the identity on $\Omega, \theta_{s+t}=\theta_{t}^{\circ} \theta_{s}$ for all $t, s \in \mathbb{R}$ and $\theta_{t} \mathbb{P}=\mathbb{P}$ for all $t \in \mathbb{R}$.

Definition 2.2.([12,13,18]) A non-autonomous random dynamical system (NRDS) $(\varphi, \theta)$ on $X$ over a metric dynamical system $\left(\Omega, \mathcal{F}, \mathbb{P},\left(\theta_{t}\right)_{t \in \mathbb{R}}\right)$ is a mapping

$$
\varphi(t, \tau, \omega): X \rightarrow X, \quad(t, \tau, \omega, x) \rightarrow \varphi(t, \tau, \omega) x,
$$

which represents the dynamics in the state space $X$ and satisfies the properties

(i) $\varphi(\tau, \tau, \omega)$ is the identity on $X$;

(ii) $\varphi(t, \tau, \omega)=\varphi\left(t, s, \theta_{s-\tau} \omega\right) \varphi(s, \tau, \omega)$ for all $\tau \leq s \leq t$;

(iii) $\omega \rightarrow \varphi(t, \tau, \omega) x$ is $\mathcal{F}$-measurable for all $t \geq \tau$ and $x \in X$.

In the sequel, we use $\mathcal{D}$ to denote a collection of some families of nonempty bounded subsets of $\mathrm{X}:$

$$
D^{\prime} \in \mathcal{D}, D^{\prime}=\{D(t, \omega) \in \mathcal{B}(X): t \in \mathbb{R}, \omega \in \Omega\} .
$$

Definition 2.3.([12,13,18]) $A$ set $B^{\prime} \in \mathcal{D}$ is called a random $\mathcal{D}$-pullback bounded absorbing set for NRDS $(\varphi, \theta)$ iffor any $t \in \mathbb{R}$ and any $D^{\prime} \in \mathcal{D}$, there exists $\tau_{0}\left(t, D^{\prime}\right)$ such that

$$
\varphi\left(t, \tau, \theta_{\tau-t} \omega\right) D\left(\tau, \theta_{\tau-t} \omega\right) \subset B(t, \omega) \text { for any } \tau \leq \tau_{0} .
$$

Definition 2.4.([12,13,18]) A set $\mathcal{A}=\{A(t, \omega): t \in \mathbb{R}, \omega \in \Omega\}$ is called a random $\mathcal{D}$-pullback attractor for $\{\varphi, \theta\}$ if the following hold:

(i) $A(t, \omega)$ is a random compact set;

(ii) $\mathcal{A}$ is invariant; that is, for $\mathbb{P}$-a.s. $\omega \in \Omega$, and $\tau \leq t, \varphi(t, \tau, \omega) A(\tau, \omega)=A\left(t, \theta_{t-\tau} \omega\right)$; 
(iii) $\mathcal{A}$ attracts all set in $\mathcal{D}$; that is, for all $B^{\prime} \in \mathcal{D}$ and $\mathbb{P}$-a.s. $\omega \in \Omega$,

$$
\lim _{\tau \rightarrow-\infty} d\left(\varphi\left(t, \tau, \theta_{\tau-t} \omega\right) B\left(\tau, \theta_{\tau-t} \omega\right), A(t, \omega)\right)=0 \text {. }
$$

Where $d$ is the Hausdorff semimetric given by $\operatorname{dist}(B, \mathcal{A})=\operatorname{supinf}_{b \in B}\|b-a\|_{X}$.

Definition 2.5.([14, 17]) A NRDS $(\varphi, \theta)$ on a Banach space $X$ is said to be pullback flattening if for every random bounded set $B^{\prime}=\{B(t, \omega): t \in \mathbb{R}, \omega \in \Omega\} \in \mathcal{D}$, for any $\varepsilon>0$ and $\omega \in \Omega$ there exists a $T\left(B^{\prime}, \varepsilon, \omega\right)<t$ and a finite dimensional subspace $X_{\epsilon}$ such that

(i) $P\left(\bigcup_{\tau \leq T_{\varepsilon}} \varphi\left(t, \tau, \theta_{\tau-t} \omega\right) B\left(\tau, \theta_{\tau-t} \omega\right)\right)$ is bounded, and

(ii) $\left\|(I-P)\left(\bigcup_{\tau \leq T_{\varepsilon}} \varphi\left(t, \tau, \theta_{\tau-t} \omega\right) B\left(\tau, \theta_{\tau-t} \omega\right)\right)\right\|_{X}<\varepsilon$,

where $P: X \rightarrow X_{\varepsilon}$ is a bounded projector.

Theorem 2.1.([14, 17]) Suppose that $(\varphi, \theta)$ is a continuous NRDS on a uniformly convex Banach space $X$.If $(\varphi, \theta)$ possesses a random $\mathcal{D}$-pullback bounded absorbing sets $B^{\prime}=\{B(t, \omega): t \in \mathbb{R}, \omega \in \Omega\}$ and $(\varphi, \theta)$ is pullback flattening, then there exists a random $\mathcal{D}$-pullback attractor $\mathcal{A}=\{A(t, \omega): t \in \mathbb{R}, \omega \in \Omega\}$.

\section{Random pullback attractor for modified Swift-Hohenberg}

In this section, we will use abstract theory in section 2 to obtain the random $\mathcal{D}$-pullback attractor for equation (1.3)-(1.5). First we introduce an Ornstein-Uhlenbeck process,

$$
z\left(\theta_{t}(\omega)\right):=-\int_{-\infty}^{0} e^{\tau}\left(\theta_{t} \omega\right)(\tau) d \tau, t \in \mathbb{R} .
$$

We known from [6], it is the solution of Langevin equation

$$
d z+z d t=d W(t)
$$

$W(t)$ is a two-sided real-valued Wiener process on a probability space $(\Omega, \mathcal{F}, \mathbb{P})$, where

$$
\Omega=\{\omega \in C(\mathbb{R}, \mathbb{R}): \omega(0)=0\},
$$

$\mathcal{F}$ is the Borel algebra induced by the compact open topology of $\Omega$, and $\mathbb{P}$ is the corresponding Wiener measure on $\{\Omega, \mathcal{F}\}$. We identify $\omega(t)$ with $W(t)$, i.e.,

Define the Wiener time shift by

$$
W(t)=W(t, \omega)=\omega(t), t \in \mathbb{R} \text {. }
$$

$$
\theta_{t} \omega(s)=\omega(s+t)-\omega(t), \omega \in \Omega, t, s \in \mathbb{R} .
$$

Then $\left(\Omega, \mathcal{F}, \mathbb{P}, \theta_{t}\right)$ is an ergodic metric dynamical system.

From [15,16,20], it is known that the random variable $z(\omega)$ is tempered and there exists a $\theta_{t}$-invariant set of full measure $\tilde{\Omega} \subset \Omega$ such that for all $\omega \in \tilde{\Omega}$ :

$$
\lim _{t \rightarrow \pm \infty} \frac{\left|z\left(\theta_{t} \omega\right)\right|}{|t|}=0, \quad \lim _{t \rightarrow \pm \infty} \frac{1}{t} \int_{0}^{t} z\left(\theta_{s} \omega\right) d s=0,
$$

and for any $\varepsilon>0$, there exists $\rho(\varepsilon)>0$, such that

$$
\left|z\left(\theta_{t} \omega\right)\right| \leq \rho(\omega)+\varepsilon|t|,\left|\int_{0}^{t} z\left(\theta_{s} \omega\right) d s\right| \leq \rho(\omega)+\varepsilon|t| .
$$

Let $v(s, \tau)=e^{-l z\left(\theta_{s-t} \omega\right)} u(s)$, then $d v=-l e^{-l z\left(\theta_{s-t} \omega\right)} u(s) d z+e^{-l z\left(\theta_{s-t} \omega\right)} d u$. Using Langevin equation, combined with the original equation (1.3), we get

$$
\begin{aligned}
& \frac{d v}{d s}+\Delta^{2} v+2 v_{x x}+(a-l z) v+b e^{l z\left(\theta_{s-t} \omega\right)}\left|v_{x}\right|^{2}+e^{2 l z\left(\theta_{s-t} \omega\right)} v^{3}=e^{-l z\left(\theta_{s-t} \omega\right)} g(x, s), \text { in } D \times[\tau, \infty), \\
& v=\Delta v=0 \text { on } \partial D \times[\tau, \infty), \\
& v(x, \tau)=v_{\tau}=e^{-l z\left(\theta_{s-t} \omega\right)} u_{\tau}(x) \text { in } D .
\end{aligned}
$$


Equation (1.3)-(1.5) are equivalent to equation (3.3)-(3.5), by a standard method, it can be proved that the problem (3.3)-(3.5) is well posed in $H_{0}^{2}(D)$, that is, for every $\tau \in \mathbb{R}$ and $v_{\tau} \in H_{0}^{2}(D)$, there exits a unique solution $v \in C\left([\tau, \infty), H_{0}^{2}(D)\right)$ (see e.g.[2,8,22]). Furthermore, the solution is continuous with respect to the initial condition $v_{\tau}$ in $H_{0}^{2}(D)$. To construct a non-autonomous random dynamical system $\{V(t, \tau, \omega)\}$ for problem (3.3)-(3.5), we define $V(t, \tau, \omega): H_{0}^{2}(D) \rightarrow H_{0}^{2}(D)$ by $V(t, \tau, \omega) v_{\tau}$. Then the system $\{V(t, \tau, \omega)\}$ is a non-autonomous random dynamical system in $H_{0}^{2}(D)$.

We now apply abstract theory in Section 2 to obtain the random $\mathcal{D}$-pullback attractors for non-autonomous modified Swift-Hohenberg equation, by the equivalent, we only consider the random $\mathcal{D}$-pullback attractor of equation (3.3)-(3.5).

For convenience, the $L^{p}(D)$ norm of $u$ will be denoted by $\|\cdot\|_{p}, H=L^{2}(D)$ with a scalar product and the norm of Sobolev spaces $W_{p}^{k}(D)$ by $\|\cdot\|_{k, p}$, we regard the space $H_{0}^{2}(D)$ endowed with the norm $\|u\|_{2,2}=\|\Delta u\|, c$ or $c(\omega)$ denote the arbitrary positive constants, which only depend on $\omega$ and may be different from line to line and even in the same line.

For our purpose that the following Gagliardo-Nirenberg inequality will be used.

Lemma 3.1. (Gagliardo-Nirenberg Inequality). Let $D$ be an open, bounded domain of the Lipschitz class in $\mathbb{R}^{n}$. Assume that $1 \leq p \leq \infty, 1 \leq q \leq \infty, 1 \leq r, 0<\theta \leq 1$ and let

$$
k-\frac{n}{p} \leq \theta\left(m-\frac{n}{q}\right)+(1-\theta) \frac{n}{r} \text {. }
$$

Then the following inequality holds:

$$
\|u\|_{k, p} \leq c(D)\|u\|_{r}^{1-\theta}\|u\|_{m, q}^{\theta}
$$

Lemma 3.2. For all $t \geq \tau$, the following inequality hold:

$$
\left\|v\left(t, \tau, \theta_{\tau-t} \omega\right)\right\|^{2} \leq c(\omega)\left(e^{-\beta(t-\tau)}\left\|v_{\tau}\right\|^{2}+1+e^{-\beta t} H(t)\right)
$$

and

$$
\int_{\tau}^{t} e^{2 \beta(s-t)+2 l \int_{s}^{t} z\left(\theta_{t-t} \omega\right) d r}\left\|v_{x x}\right\|^{2} d s \leq c(\omega)\left(e^{-\beta(t-\tau)}\left\|v_{\tau}\right\|^{2}+1+e^{-\beta t} H(t)\right) .
$$

Proof. Let $v(s)$ or $v$ denotes $v\left(s, \tau, \theta_{s-t} \omega\right)$ be the solution of equation (3.3)-(3.5). Taking the inner product of equation (3.3) with $v$, we get

$$
\begin{aligned}
& \frac{1}{2} \frac{d}{d s}\|v\|^{2}+\|\Delta v\|^{2}+2(\Delta v, v)+(a-l z)\|v\|^{2}+b e^{l z\left(\theta_{s-t} \omega\right)}\left(v_{x}^{2}, v\right)\|v\|_{4}^{4} \\
+ & e^{2 l z\left(\theta_{s-t} \omega\right)}\|v\|_{4}^{4}=e^{-l z\left(\theta_{s-t} \omega\right)}(g(x, s), v) .
\end{aligned}
$$

Using Young inequality, we get

$$
|(2 \Delta v, v)| \leq \frac{1}{4}\|\Delta v\|^{2}+4\|v\|^{2}
$$

By integration by parts, we obtain

$$
b e^{l z\left(\theta_{s-t} \omega\right)}\left(v_{x}^{2}, v\right)=b e^{l z\left(\theta_{s-t} \omega\right)} \int_{D} v_{x}^{2} v d x=-b e^{l z\left(\theta_{s-t} \omega\right)} \int_{D}\left(v^{2} v_{x x}+v v_{x}^{2}\right) d x,
$$

thus

$$
b e^{l z\left(\theta_{s-t} \omega\right)}\left(v_{x}^{2}, v\right)=-\frac{b}{2} e^{l z\left(\theta_{s-t} \omega\right)} \int_{D} v^{2} v_{x x} d x .
$$

Applying the $\mathrm{H}$ ö lder inequality and Young inequality, we get

$$
b e^{l z\left(\theta_{s-t} \omega\right)}\left|\left(v_{x}^{2}, v\right)\right|=\left|\frac{b}{2} e^{l z\left(\theta_{s-t} \omega\right)} \int_{D} v^{2} v_{x x} d x\right| \leq\left|\frac{b}{2}\right| e^{l z\left(\theta_{s-t} \omega\right)}\left\|v_{x x}\right\|\|v\|_{4}^{2} \leq \eta\left\|v_{x x}\right\|^{2}+\frac{b^{2}}{16 \eta} e^{2 l z\left(\theta_{s-t} \omega\right)}\|v\|_{4}^{4},
$$


and

$$
e^{-l z\left(\theta_{s-t} \omega\right)}|(g(x, s), v)| \leq\|v\|^{2}+\frac{1}{4} e^{-2 l z\left(\theta_{s-t} \omega\right)} \|\left(g(x, s) \|^{2}\right.
$$

For convenience, we take $\eta=\frac{1}{4},|b|<2(|b|<4$, the same conclusion hold), we obtain

$$
\frac{d}{d s}\|v\|^{2}+\|\Delta v\|^{2}+2(a-l z-5)\|v\|^{2}+2\left(1-\frac{b^{2}}{4}\right) e^{2 l z\left(\theta_{s-t} \omega\right)}\|v\|_{4}^{4} \leq \frac{1}{2} e^{-2 l z\left(\theta_{s-t} \omega\right)} \|\left(g(x, s) \|^{2} .\right.
$$

Taking $\beta>0$ such that $a-\beta-5<0$, we have

$$
\begin{aligned}
& \frac{d}{d s}\|v\|^{2}+\|\Delta v\|^{2}+2(\beta-l z)\|v\|^{2}+2\left(1-\frac{b^{2}}{4}\right) e^{2 l z\left(\theta_{s-t} \omega\right)}\|v\|_{4}^{4} \\
\leq & -2(a-\beta-5)\|v\|^{2}+\frac{1}{2} e^{-2 l z\left(\theta_{s-t}(\omega)\right.} \|\left(g(x, s) \|^{2} .\right.
\end{aligned}
$$

By the Sobolev imbedding $L^{4}(D) \subset L^{2}(D)$ and Young inequality, we get

$$
-2(a-\beta-5)\|v\|^{2} \leq c\|v\|_{4}^{2} \leq 2\left(1-\frac{b^{2}}{4}\right) e^{2 l z\left(\theta_{s-t} \omega\right)}\|v\|_{4}^{4}+c e^{-2 l z\left(\theta_{s-t} \omega\right)} .
$$

Thus we obtain

$$
\frac{d}{d s}\|v\|^{2}+\|\Delta v\|^{2}+2(\beta-l z)\|v\|^{2} \leq c e^{-2 l z\left(\theta_{s-t}(\omega)\right.}\left(1+\|\left(g(x, s) \|^{2}\right) .\right.
$$

Multiply this by $e^{2 \beta s-2 l \int_{\tau}^{s} z\left(\theta_{r-t} \omega\right) d r}$ and integrating from $\tau$ to $t$, we have

$$
\begin{aligned}
& \|v(t)\|^{2}+\int_{\tau}^{t} e^{2 \beta(s-t)+2 l \int_{s}^{t} z\left(\theta_{r-t}(\omega) d r\right.}\|\Delta v\|^{2} d s \\
\leq & e^{-2 \beta(t-\tau)+2 l \int_{\tau}^{t} z\left(\theta_{r-t} \omega\right) d r}\left\|v_{\tau}\right\|^{2}+\int_{\tau}^{t} e^{-2 \beta(t-s)+2 l \int_{s}^{t} z\left(\theta_{r-t}(\omega)-2 l z\left(\theta_{s-t}(\omega)\right.\right.}\left(1+\|g(x, s)\|^{2}\right) d s \\
\leq & e^{-2 \beta(t-\tau)+2 l \int_{\tau-t}^{0} z\left(\theta_{r} \omega\right) d r}\left\|v_{\tau}\right\|^{2}+\int_{\tau}^{t} e^{-2 \beta(t-s)+2 l \int_{s-t}^{0} z\left(\theta_{r}(\omega)-2 l z\left(\theta_{s-t}(\omega)\right.\right.}\left(1+\|g(x, s)\|^{2}\right) d s
\end{aligned}
$$

From (3.2), we get

$$
2 l \int_{\tau-t}^{0} z\left(\theta_{r} \omega\right) d r \leq \rho(\omega)+\beta(t-\tau), 2 l \int_{s-t}^{0} z\left(\theta_{r} \omega\right)-2 l z\left(\theta_{s-t} \omega\right) \leq \rho(\omega)+\beta(t-s) .
$$

Then we have

$$
\begin{gathered}
\quad\|v(t)\|^{2}+\int_{\tau}^{t} e^{2 \beta(s-t)+2 l \int_{s}^{t} z\left(\theta_{r-t}(\omega) d r\right.}\|\Delta v\|^{2} d s \\
\leq c(\omega)\left(e^{-\beta(t-\tau)}\left\|v_{\tau}\right\|^{2}+\int_{\tau}^{t} e^{-\beta(t-s)}\left(1+\|g(x, s)\|^{2}\right) d s\right) \\
\leq c(\omega)\left(e^{-\beta(t-\tau)}\left\|v_{\tau}\right\|^{2}+1+e^{-\beta t} \int_{\tau}^{t} e^{\beta s}\|g(x, s)\|^{2} d s\right) .
\end{gathered}
$$

Thus we get the desired results.

Lemma 3.3. For all $t \geq \tau$, the following inequality hold:

$$
\begin{aligned}
\left\|\Delta v\left(t, \tau, \theta_{\tau-t} \omega\right)\right\|^{2} & \leq c(\omega)\left[\left(1+\frac{1}{t-\tau}\right) e^{-\beta(t-\tau)}\left\|v_{\tau}\right\|^{2}+e^{-\frac{11}{3} \beta(t-\tau)}\left\|v_{\tau}\right\|^{\frac{22}{3}}\right. \\
& \left.+e^{-\beta t}\left(H(t)+\int_{-\infty}^{t} e^{-\frac{8}{3} \beta s} H^{\frac{11}{3}}(s) d s\right)\right]
\end{aligned}
$$

Proof. Taking inner product of equation (3.3) with $\Delta^{2} v$, we have

$$
\begin{aligned}
& \frac{1}{2} \frac{d}{d s}\|\Delta v\|^{2}+\left\|\Delta^{2} v\right\|^{2}+2\left(\Delta v, \Delta^{2} v\right)+(a-l z)\left\|_{\Delta v}\right\|^{2} \\
+ & b e^{l z\left(\theta_{s-t} \omega\right)}\left(v_{x}^{2}, \Delta^{2} v\right)+e^{2 l z\left(\theta_{s-t} \omega\right)}\left(v^{3}, \Delta^{2} v\right)=e^{-l z\left(\theta_{s-t} \omega\right)}\left(g(x, s), \Delta^{2} v\right) .
\end{aligned}
$$


By the Hölder inequality, Young inequality and Gagliardo-Nirenberg inequality, we get

$$
\begin{gathered}
-2\left(\Delta v, \Delta^{2} v\right) \leq \frac{1}{8}\left\|\Delta^{2} v\right\|^{2}+8\|\Delta v\|^{2}, \\
|b| e^{l z\left(\theta_{s-t}(\omega)\right.}\left|\left(v_{x}^{2}, \Delta^{2} v\right)\right| \leq|b| e^{l z\left(\theta_{s-t}(\omega)\right.}\left\|v_{x}\right\|_{4}^{2}\left\|\Delta^{2} v\right\| \leq c e^{l z\left(\theta_{s-t}(\omega)\right.}\|v\|^{\frac{11}{8}}\left\|\Delta^{2} v\right\|^{\frac{13}{8}} \\
\leq \frac{1}{8}\left\|\Delta^{2} v\right\|^{2}+c e^{\frac{16}{3} l z\left(\theta_{s-t}(\omega)\right.}\|v\|^{\frac{22}{3}}, \\
e^{2 l z\left(\theta_{s-t}(\omega)\right.}\left|\left(v^{3}, \Delta^{2} v\right)\right| \leq e^{2 l z\left(\theta_{s-t} \omega\right)}\|v\|_{6}^{3}\left\|\Delta^{2} v\right\| \leq c e^{2 l z\left(\theta_{s-t} \omega\right)}\left\|\Delta^{2} v\right\|\|v\|^{11}\left\|\Delta^{2} v\right\|^{\frac{1}{4}} \\
=c e^{2 l z\left(\theta_{s-t} \omega\right)}\left\|\Delta^{2} v\right\|^{\frac{5}{4}}\|v\|^{\frac{11}{4}} \leq \frac{1}{8}\left\|\Delta^{2} v\right\|^{2}+c e^{\frac{16}{3} l z\left(\theta_{s-t}(\omega)\right.}\|v\|^{\frac{22}{3}} \\
e^{-l z\left(\theta_{s-t}(\omega)\right.}\left(g(x, s), \Delta^{2} v\right) \leq \frac{1}{8}\left\|\Delta^{2} v\right\|^{2}+2 e^{-2 l z\left(\theta_{s-t}(\omega)\right.}\|g(x, s)\|^{2} .
\end{gathered}
$$

Putting all these inequalities together, we deduce

$$
\frac{d}{d s}\|\Delta v\|^{2}+2(\beta-l z)\left\|_{\Delta v}\right\|^{2} \leq 2(8+\beta-a)\|\Delta v\|^{2}+c\left(e^{\frac{16}{3} l z\left(\theta_{s-t}(\omega)\right.}\|v\|^{\frac{22}{3}}+e^{-2 l z\left(\theta_{s-t}(\omega)\right.}\|g(x, s)\|^{2}\right) .
$$

Multiplying this by $(s-\tau) e^{2 \beta s-2 l \int_{\tau}^{s} z\left(\theta_{r-t} \omega\right) d r}$ and integrating it over $(\tau, t)$, we get

$$
\begin{gathered}
(t-\tau) e^{2 \beta t-2 l \int_{\tau}^{t} z\left(\theta_{r-t}(\omega) d r\right.}\|\Delta v(t)\|^{2} \leq c\left[\int_{\tau}^{t} e^{2 \beta s-2 l \int_{\tau}^{s} z\left(\theta_{r-t} \omega\right) d r}\|\Delta v\|^{2} d s\right. \\
\left.+\int_{\tau}^{t}(s-\tau) e^{2 \beta s-2 l \int_{\tau}^{s} z\left(\theta_{r-t} \omega\right) d r}\left(\|\Delta v\|^{2}+e^{\frac{16}{3} l z\left(\theta_{s-t}(\omega)\right.}\|v\|^{\frac{22}{3}}+e^{-2 l z\left(\theta_{s-t} \omega\right)}\|g(x, s)\|^{2}\right) d s\right] .
\end{gathered}
$$

Then we have

$$
\begin{gathered}
\|\Delta v(t)\|^{2} \leq c\left[\left(1+\frac{1}{t-\tau}\right) \int_{\tau}^{t} e^{-2 \beta(t-s)+2 l \int_{s}^{t} z\left(\theta_{r-t} \omega\right) d r}\|\Delta v\|^{2} d s\right. \\
\left.+\int_{\tau}^{t} e^{-2 \beta(t-s)+2 l \int_{s}^{t} z\left(\theta_{r-t} \omega\right) d r+\frac{16}{3} l z\left(\theta_{s-t} \omega\right)}\|v\|^{\frac{22}{3}} d s+\int_{\tau}^{t} e^{-2 \beta(t-s)+2 l \int_{s}^{t} z\left(\theta_{r-t} \omega\right) d r-2 l z\left(\theta_{s-t} \omega\right)}\|g(x, s)\|^{2}\right] .
\end{gathered}
$$

By (3.2), (3.6) and the inequality $(a+b)^{r} \leq c\left(a^{r}+b^{r}\right)(a, b>0, r \geq 1)$, we get

$$
\begin{aligned}
& \int_{\tau}^{t} e^{-2 \beta(t-s)+2 l \int_{s}^{t} z\left(\theta_{r-t}(\omega) d r+\frac{16}{3} l\left(\theta_{s-t} \omega\right)\right.}\|v\|^{\frac{22}{3}} d s \leq c(\omega) \int_{\tau}^{t} e^{-\beta(t-s)}\|v\|^{\frac{22}{3}} d s \\
\leq & c(\omega) e^{-\beta t} \int_{\tau}^{t} e^{\beta s}\left(e^{-\beta(s-\tau)}\left\|v_{\tau}\right\|^{2}+1+e^{-\beta s} H(s)\right)^{\frac{11}{3}} d s \\
\leq & c(\omega) e^{-\beta t} \int_{\tau}^{t} e^{\beta s}\left(e^{-\frac{11}{3} \beta(s-\tau)}\left\|v_{\tau}\right\|^{\frac{22}{3}}+1+e^{-\frac{11}{3} \beta s} H^{\frac{11}{3}}(s)\right) d s \\
\leq & c(\omega)\left(e^{-\frac{11}{3} \beta(t-\tau)}\left\|v_{\tau}\right\|^{\frac{22}{3}}+1+e^{-\beta t} \int_{\tau}^{t} e^{-\frac{8}{3} \beta s} H^{\frac{11}{3}}(s) d s\right) .
\end{aligned}
$$

Thus we have

$$
\begin{aligned}
\|\Delta v(t, \tau)\|^{2} \leq & c(\omega)\left[\left(1+\frac{1}{t-\tau}\right) e^{-\beta(t-s)}\left\|v_{\tau}\right\|^{2}+e^{-\frac{11}{3} \beta(t-\tau)}\left\|v_{\tau}\right\|^{\frac{22}{3}}+1\right. \\
& \left.\left.+e^{-\beta t}\left(H(t)+\int_{-\infty}^{t} e^{-\frac{8}{3} \beta s} H^{\frac{11}{3}}(s)\right) d s\right)\right] .
\end{aligned}
$$

We complete the proof of Lemma 3.3.

Let $\mathcal{R}$ be the set of all function $r: \mathbb{R} \rightarrow(0,+\infty)$ such that $\lim _{t \rightarrow-\infty} e^{\beta t} r^{2}(t)=0$ and denote by $\mathcal{D}$ the class of all families $\hat{D}=\{D(t): t \in \mathbb{R}\}$ such that $D(t) \subset \bar{B}(r(t))$ for some $r(t) \in \mathcal{R}, \bar{B}(r(t))$ denote the closed ball in $H_{0}^{2}(D)$ with radius $r(t)$. Let 


$$
r_{1}^{2}(t)=2 c(\omega)\left[1+e^{-\beta t}\left(H(t)+\int_{-\infty}^{t} e^{-\frac{8}{3} \beta s} H^{\frac{11}{3}}(s) d s\right)\right]
$$

By lemma 3.3 for any $\hat{D} \in \mathcal{D}$ and $t \in \mathbb{R}$, there exists $\tau_{0}(\hat{D}, t, \omega)<t$ such that

$$
\left\|\Delta v\left(t, \tau, \theta_{\tau-t} \omega\right)\right\| \leq r_{1}(t) \text {, for any } \tau<\tau_{0} \text {. }
$$

Since $0 \leq \gamma<\frac{3 \beta}{11}$, simple calculation imply that $r_{1}(t) \in \mathcal{R}$, which say that the $\bar{B}\left(r_{1}(t)\right)$ be a family of random $\mathcal{D}$-pullback bounded absorbing sets in $H_{0}^{2}(D)$ and $\left\{\bar{B}\left(r_{1}(t)\right)\right\} \in \mathcal{D}$.

Theorem 3.1. The non-autonomous random dynamical system to problem (1.1)-(1.3) possesses a unique random $\mathcal{D}$-pullback attractor in $H_{0}^{2}(D)$.

Proof. We need only prove that the dynamical system (3.3)-(3.5) satisfies the pullback flattening condition. Since $A^{-1}$ is a continuous compact operator in $H_{0}^{2}(D)$, by the classical spectral theorem, there exists a sequence $\left\{\lambda_{j}\right\}_{j=1}^{\infty}$ satisfing

$$
0<\lambda_{1} \leq \lambda_{2} \leq \cdots \lambda_{j} \leq \cdots, \lambda_{j} \rightarrow+\infty, \text { as } j \rightarrow+\infty,
$$

and a family of elements $\left\{e_{j}\right\}_{j=1}^{\infty}$ of $H_{0}^{2}(D)$ which are orthonormal in $H$ such that

$$
A e_{j}=\lambda_{j} e_{j}, \forall j \in \mathbb{N}^{+} \text {. }
$$

Let $H_{m}=\operatorname{span}\left\{e_{1}, e_{2}, \cdots, e_{m}\right\}$ in $H$ and $P_{m}: H \rightarrow H_{m}$ be an orthogonal projector. For any $v \in H$ we write

$$
v=P_{m} v+\left(I-P_{m}\right) v=v_{1}+v_{2} .
$$

Taking inner product of (3.3) with $\Delta^{2} v_{2}$ in $H$, we get

$$
\begin{aligned}
& \frac{1}{2} \frac{d}{d s}\left\|\Delta v_{2}\right\|^{2}+\left\|\Delta^{2} v_{2}\right\|^{2}+2\left(\Delta v, \Delta^{2} v_{2}\right)+(a-l z)\left\|_{\Delta v_{2}}\right\|^{2} \\
+ & b e^{l z\left(\theta_{s-t} \omega\right)}\left(|\nabla v|^{2}, \Delta^{2} v_{2}\right)+e^{2 l z\left(\theta_{s-t} \omega\right)}\left(v^{3}, \Delta^{2} v_{2}\right)=e^{-l z\left(\theta_{s-t} \omega\right)}\left(g(x, s), \Delta^{2} v_{2}\right) .
\end{aligned}
$$

By the Hölder inequality, Young inequality and Gagliardo-Nirenberg inequality, we get

$$
\begin{aligned}
-2(\Delta v & \left.\Delta^{2} v_{2}\right) \leq \frac{1}{8}\left\|\Delta^{2} v_{2}\right\|^{2}+8\|\Delta v\|^{2}, \\
-b e^{l z\left(\theta_{s-t} \omega\right)}\left(v_{x}^{2}, \Delta^{2} v_{2}\right) & \leq \frac{1}{8}\left\|\Delta^{2} v_{2}\right\|^{2}+2 b^{2} e^{2 l z\left(\theta_{s-t} \omega\right)}\left\|v_{x}\right\|_{4}^{4} \\
& \leq \frac{1}{8}\left\|\Delta^{2} v_{2}\right\|^{2}+c e^{2 l z\left(\theta_{s-t} \omega\right)}\|v\|^{4(1-\theta)}\|\Delta v\|^{4 \theta} \\
& =\frac{1}{8}\left\|\Delta^{2} v_{2}\right\|^{2}+c e^{2 l z\left(\theta_{s-t} \omega\right)}\|v\|^{3}\|\Delta v\| \quad\left(\theta=\frac{1}{4}\right) \\
& \leq \frac{1}{8}\left\|\Delta^{2} v_{2}\right\|^{2}+\frac{1}{2}\|\Delta v\|^{2}+c e^{4 l z\left(\theta_{s-t} \omega\right)}\|v\|^{6},
\end{aligned}
$$




$$
\begin{aligned}
-e^{2 l z\left(\theta_{s-t} \omega\right)}\left(v^{3}, \Delta^{2} v_{2}\right) & \leq \frac{1}{8}\left\|\Delta^{2} v_{2}\right\|^{2}+2 e^{4 l z\left(\theta_{s-t} \omega\right)}\|v\|_{6}^{3} \\
& \leq \frac{1}{8}\left\|\Delta^{2} v_{2}\right\|^{2}+c e^{4 l z\left(\theta_{s-t} \omega\right)}\|v\|^{3(1-\theta)}\|\Delta v\|^{3 \theta} \\
& =\frac{1}{8}\left\|\Delta^{2} v_{2}\right\|^{2}+c e^{4 l z\left(\theta_{s-t}(\omega)\right.}\|v\|^{2}\|\Delta v\| \quad\left(\theta=\frac{1}{3}\right) \\
& \leq \frac{1}{8}\left\|\Delta^{2} v_{2}\right\|^{2}+\frac{1}{2}\|\Delta v\|^{2}+c e^{8 l z\left(\theta_{s-t} \omega\right)}\|v\|^{4}, \\
e^{-l z\left(\theta_{s-t} \omega\right)}\left(g(x, s), \Delta^{2} v_{2}\right) & \leq \frac{1}{8}\left\|\Delta^{2} v_{2}\right\|^{2}+2 e^{-2 l z\left(\theta_{s-t} \omega\right)}\|g(x, s)\|^{2} .
\end{aligned}
$$

Putting all these inequalities together, we have

$$
\begin{aligned}
& \frac{d}{d s}\left\|\Delta v_{2}\right\|^{2}+\left\|\Delta v_{2}^{2}\right\|^{2}+2(a-l z)\left\|\Delta v_{2}\right\|^{2} \\
\leq & 18\|\Delta v\|^{2}+c\left(e^{4 l z\left(\theta_{s-t} \omega\right)}\|v\|^{6}+e^{8 l z\left(\theta_{s-t} \omega\right)}\|v\|^{4}+e^{-2 l z\left(\theta_{s-t}(\omega)\right.}\|g(x, s)\|^{2}\right) .
\end{aligned}
$$

$\lambda_{n}\left\|\Delta v_{2}\right\|^{2} \leq\left\|\Delta^{2} v_{2}\right\|^{2}$, which imply that

$$
\begin{aligned}
& \frac{d}{d s}\left\|\Delta v_{2}\right\|^{2}+\left(\lambda_{n}-2 l z\right)\left\|\Delta v_{2}\right\|^{2} \\
\leq & c\left(\|\Delta v\|^{2}+e^{4 l z\left(\theta_{s-t}(\omega)\right.}\|v\|^{6}+e^{8 l z\left(\theta_{s-t} \omega\right)}\|v\|^{4}+e^{-2 l z\left(\theta_{s-t}(\omega)\right.}\|g(x, s)\|^{2}\right) .
\end{aligned}
$$

Multiply this by $(s-\tau) e^{\lambda_{n} s-2 l \int_{\tau}^{s} z\left(\theta_{r-t}(\omega) d r\right.}$ and integrating from $\tau$ to $t$, we obtain

$$
\begin{gathered}
(t-\tau) e^{\lambda_{n} t-2 l \int_{\tau}^{t} z\left(\theta_{r-t} \omega\right) d r}\left\|\Delta v_{2}\right\|^{2} \leq c\left[\int_{\tau}^{t}(1+s-\tau) e^{\lambda_{n} s-2 l \int_{\tau}^{s} z\left(\theta_{r-t} \omega\right) d r}\|\Delta v\|^{2} d s\right. \\
\left.+\int_{\tau}^{t}(s-\tau) e^{\lambda_{n} s-2 l \int_{\tau}^{s} z\left(\theta_{r-1} \omega\right) d r}\left(e^{4 l z\left(\theta_{s-t} \omega\right)}\|v\|^{6}+e^{8 l z\left(\theta_{s-t} \omega\right)}\|v\|^{4}+e^{-2 l z\left(\theta_{s-t}(\omega)\right.}\|g(x, s)\|^{2}\right) d s\right]
\end{gathered}
$$

Thus we get

$$
\begin{aligned}
\left\|\Delta v_{2}\right\|^{2} & \leq c\left[\left(1+\frac{1}{t-\tau}\right) \int_{\tau}^{t} e^{\lambda_{n}(s-t)+2 l \int_{s}^{t} z\left(\theta_{r-t} \omega\right) d r}\|\Delta v\|^{2} d s+\int_{\tau}^{t} e^{\lambda_{n}(s-t)+2 l \int_{s}^{t} z\left(\theta_{r-t} \omega\right) d r+4 l z\left(\theta_{s-t}(\omega)\right.}\|v\|^{6} d s\right. \\
& +\int_{\tau}^{t} e^{\lambda_{n}(s-t)+2 l \int_{s}^{t} z\left(\theta_{r-t}(\omega) d r+8 l z\left(\theta_{s-t}(\omega)\right.\right.}\|v\|^{4} d s+\int_{\tau}^{t} e^{\lambda_{n}(s-t)+2 l \int_{s}^{t} z\left(\theta_{r-t} \omega\right) d r-2 l z\left(\theta_{s-t} \omega\right)}\|g(x, s)\|^{2} d s \\
& \leq c(\omega)\left[\left(1+\frac{1}{t-\tau}\right) \int_{\tau}^{t} e^{\left(\lambda_{n}-\beta\right)(s-t)}\|\Delta v\|^{2} d s+\int_{\tau}^{t} e^{\left(\lambda_{n}-\beta\right)(s-t)}\|v\|^{6} d s\right. \\
& \left.+\int_{\tau}^{t} e^{\left(\lambda_{n}-\beta\right)(s-t)}\|v\|^{4} d s+\int_{\tau}^{t} e^{\left(\lambda_{n}-\beta\right)(s-t)}\|g(x, s)\|^{2} d s\right] \\
& \left.=c(\omega)\left(I_{1}+I_{2}+I_{3}+I_{4}\right)\right) .
\end{aligned}
$$

By simple calculation, we find that there exists $N \in \mathbb{N}, \forall n>N, \lambda_{n}-\beta>\beta$, and

$$
I_{1} \leq\left(1+\frac{1}{t-\tau}\right) \int_{\tau}^{t} e^{\beta(s-t)}\|\Delta v\|^{2} d s<\infty, e^{\lambda_{n}(s-t)}\|\Delta v(s)\|^{2} \rightarrow 0 \text { as } n \rightarrow \infty,
$$

According to Lebesgue dominated convergent theorem, we obtain

$$
I_{1} \rightarrow 0 \text { as } n \rightarrow \infty \text {. }
$$

Using (3.6), we get

$$
\begin{aligned}
I_{2} & \leq c \int_{\tau}^{t} e^{\left(\lambda_{n}-\beta\right)(s-t)}\left(e^{-\beta(s-\tau)}\left\|v_{\tau}\right\|^{2}+1+e^{-\beta s} H(s)\right)^{3} d s \\
& \leq c \int_{\tau}^{t} e^{\left(\lambda_{n}-\beta\right)(s-t)}\left(e^{-3 \beta(s-\tau)}\left\|v_{\tau}\right\|^{6}+1+e^{-3 \beta s} H^{3}(s)\right) d s \\
& \leq c\left[e^{-\beta t} \frac{e^{-3 \beta(t-\tau)}}{\lambda_{n}-4 \beta}\left\|v_{\tau}\right\|^{6}+\frac{1}{\lambda_{n}-\beta}+\frac{e^{-4 \beta t}}{\lambda_{n}-4 \beta} H^{3}(t)\right] \rightarrow 0 \text { as } n \rightarrow \infty,
\end{aligned}
$$




$$
\begin{aligned}
I_{3} & \leq c \int_{\tau}^{t} e^{\left(\lambda_{n}-\beta\right)(s-t)}\left(e^{-\beta(s-\tau)}\left\|v_{\tau}\right\|^{2}+1+e^{-\beta s} H(s)\right)^{2} d s \\
& \leq c \int_{\tau}^{t} e^{\left(\lambda_{n}-\beta\right)(s-t)}\left(e^{-2 \beta(s-\tau)}\left\|v_{\tau}\right\|^{4}+1+e^{-2 \beta s} H^{2}(s)\right) d s \\
& \leq c\left[e^{-\beta t} \frac{e^{-2 \beta(t-\tau)}}{\lambda_{n}-3 \beta}\left\|v_{\tau}\right\|^{4}+\frac{1}{\lambda_{n}-\beta}+\frac{e^{-3 \beta t}}{\lambda_{n}-3 \beta} H^{2}(t)\right] \rightarrow 0, \text { as } n \rightarrow \infty,
\end{aligned}
$$

and

$$
I_{4} \leq e^{-\beta t} \int_{\tau}^{t} e^{\beta s}\|g(x, s)\|^{2} d s, e^{\left(\lambda_{n}-\beta\right)(s-t)}\|g(x, s)\|^{2} \rightarrow 0 \text { as } n \rightarrow \infty,
$$

Again using Lebesgue dominated convergent theorem, we get

$$
e^{\left(\lambda_{n}-\beta\right)(s-t)}\|g(x, s)\|^{2} d s \rightarrow 0 \text { as } n \rightarrow \infty .
$$

In summary, we obtain that the terms on the right hand of inequality (3.13) tend to 0 as $n \rightarrow \infty$, which say that $\left\|v_{2}\left(t, \tau, \theta_{\tau-t} \omega\right)\right\| \rightarrow 0$, i.e., the random dynamical system (3.3)-(3.5) satisfies pullback flattening.

\section{Conclusions}

This paper extends the existence of pullback attractor of non-autonomous modified S-H equation to the case of non-autonomous stochastic modified S-H equation with multiplicative noise. In the concrete experiment, the random term in the equation is more consistent with the actual problem. For S-H equation with multiplicative noise, the external force has exponential growth, we have proved that the equation exists a random $\mathcal{D}$-pullback attractor in one dimension. In the future work, we will continue to investigate whether the same results can be obtained when the spatial dimension is two-dimensional or n-dimensional.

Author Contributions: All the authors have equal contribution to this study. All authors have read and agreed to the published version of the manuscript.

Funding: This research was supported by the National Natural Science Foundation of China(11761044, 11661048) and the key constructive discipline of Lanzhou City University (LZCU-ZDJSXK-201706).

Conflflicts of Interest: The authors declare no conflict of interest. The funders had no role in the design of the study; in the collection, analyses, or interpretation of data; in the writing of the manuscript, or in the decision to publish the results.

\section{References}

1. J.B. Swift, P.C. Hohenberg, Hydrodynamics fluctuations at the convective instability, Phys. Rev. A, 1977, 15, 319-328.

2. D. Blömker, M.Hairer, G.Pavlioyis, Stochastic Swift-Hohenberg equation near a change of stability, Proceedings of Equa.Diff., 2005, 11, 27-37.

3. A.Doelman, B.Standstede, A.Scheel, G. Schneider, Propagation of hexagonal pat- terens near onset, European J.Appl.Math., 2003, 85-110.

4. L.A.Peletier, V. Rottscha"fer, Large time behavior of solution of the Swift-Hohenberg equation, Comptes Rendus Mathematique, 2003, 336(3), 225-230.

5. L.A. Peletier, V.Rottscha"fer, Pattern selection of solutions of the Swift-Hohenberg equation, Physica D, 2004, 194(1-2),95-126.

6. L.A. Peletier, J.F.Williamas, Some canonical bifurcations in the Swift-Hohenberg equation, SIAM J.Appl.Dyn.Syst., 2007, 6, 208-235.

7. L.Song, Y.Zhang, T.Ma,Global attractor of a modified Swift-Hohenberg equation in $\mathrm{H}^{\mathrm{k}}$ spaces, Nonlinear Anal., 2006, 64, 483-498.

8. M.Polat, Global attractor for a modified Swift-Hohenbergequation, Comput. Math. Appl., 2009,57, 62-66. 
9. S.H. Park, J.Y. Park, Pullback attractors for a non-autonomous modified Swift- Hohenberg equation, Comput. Math. Appl., 2014, 67, 542-548.

10. L.Xu, Q.Ma, Existence of the uniform attractors for a non-autonomous modified Swift-Hohenberg equation, Advances in Difference Equations, 2015, 2015(1):153.

11. C.Guo, Y.Guo, C.Li, Dynamical behavior of a local modifed stochastic Swift- Hohonberg equation with multiplicative noise, Boundary Value Problems, 2017, DOI: 10.1186/s13661-017-0753-5.

12. Y.Li, C.Zhong, Pullback attractors for the norm-to-weak continuous process and ap- plication to the non-autonomous reaction-diffusion equations, Applied Mathematics and Computation, 2007, 190, 1020-1029.

13. B.Wang, Sufficient and necessary criteria for existence of pullback attractors for non- compact random dynamical system, J.Differential Equations, 2009, 253, 544-1583.

14. P.E.Kloeden, J.A.Langa, Flattening, squeezing and the existence of random attractors, Proc.R.Soc.A, 2007, 463, 163-181.

15. P.W.Bates, K.Lu, B.Wang, Random attractors for stochastic reaction-diffusion equa- tions on unbounded domains, J.Differential Equations, 2009, 246, 845-869.

16. Y.Li, B.Guo, Random attractors for quasi-continuous random dynamical systems and application to stochastic reaction-diffiusion equation, J.Differential Equations, 2008, 245, 1775-1800.

17. Y.Li, J.Wei, T.Zhao, The existence of random D-pullback attractors for a random dynamical system and its applicatin, Journal of Applied Analysis and Computaiton, 2019, 9(4), 1571-1588.

18. Y.Wang, J.Wang, Pullback attractors for multi-valued non-compact random dynam- ical systems generated by reaction-diffusion equations on an unbounded domain, J.Differential Equations, 2015, 259, 728-776.

19. Z.Wang, S.Zhou, Random attractor for stochastic reaction-diffusion equation with multiplicative noise on unbounded domains\}, Journal of Mathematical Analysis and Applications, 2011, 384, 160-172.

20. J.Duan, An introduction to Stochastic Dynamics, Science Press, Beijing, 2014.

21. L.Arnold, Random Dynamical System, Springer-Verlag, 1998.

22. R.Temam, Infinite-dimensional Dynamical Systems in Mechanics and Physics, 2nd ed., Springer-Verlag, 1997. 